\title{
Sneaky politics
}

\author{
Scientists need to mobilize to combat changing tactics by animal rights activists.
}

$\mathbf{S}$ cientists are no strangers to the intimidation tactics employed by animal rights activists. Despite the rise of pro-animal testing movements, such as Pro-test (http://www.pro-test.org.uk/), animal rights extremism is alive and well; in April this year, animal rights activists occupied an animal research facility at the University of Milan, destroying substantial work on animal models of important psychiatric disorders. However, what is more insidious, but equally successful, in disrupting research is the increasing success of more moderate animal rights activism at influencing government policy at a local level, making it harder and harder to get approval for otherwise scientifically justifiable and important animal research. Animal rights activists have also successfully campaigned to force most commercial airlines to shut down their transport of non-human primates to and from research centers. Such campaigns, couched with the laudable goal of reducing the number of animals in research and ensuring that animals do not unduly suffer, do not get as much press attention and tend to slip under the radar of even vigilant politicians, yet they can be detrimental to the progress of research. Now, more than ever, researchers have a duty to speak up against such campaigns and make a public case for why animal research is vital for advances in science and medicine.

A good illustration of the change in tactics from direct intimidation of researchers to attempts to influence government policy is provided by the dire situation faced by primate labs in Germany. In 2007, after a concentrated campaign by animal rights activists, Bremen's Social Democrat-Green coalition state government decided not to renew a license for neuroscience studies in macaque monkeys at the local university, in keeping with their stated aim of abolishing macaque experiments. After a long legal battle, a German court ruled that local authorities were wrong to block the license (http://tinyurl.com/ cgw49z4), but the legal battle is still ongoing, with scientists and university officials investing significant time, effort and money to overturn a decision that should not have been made in the first place. In the meantime, the uncertainties involved have meant that actual research and the recruitment of new students have suffered greatly. Researchers elsewhere in Germany also share concerns about increasing government demands that make macaque work, in particular, harder, with an increasing amount of red tape involved to get ethical approval for such studies. What is especially worrying is that it is challenging to get ethical approval for studies involving the use of larger sample sizes, even when these larger numbers are scientifically justified and perhaps necessary to draw meaningful conclusions. Such a blind insistence on limiting the use of animals to an arbitrary number is ethically, as well as scientifically, dubious: the use of sample sizes too small to detect a real effect may counterintuitively lead to more animals being used overall, as multiple small, underpowered studies struggle to detect an effect and others follow up spurious results that can come out of small studies.
Nor is this phenomenon of increasingly onerous requirements to gain approval for animal experiments limited to Germany or Europe. Animal rights activists have also made inroads in the US government with the introduction of the Great Ape Protection and Cost Savings Act. This legislation aims to eliminate research with chimpanzees, arguing, among other things, that such a move would save money, a critical issue for congressional discussion these days. This is a false promise: the Congressional Budget Office analysis suggests that there would not actually be any cost savings associated with this bill (http:// tinyurl.com/bvwdhc9), but on the face of it, such a bill would appeal to the broader audience of the cost conscious, rather than just animal rights sympathizers.

In yet another example of less obvious tactics, PETA successfully lobbied to have most commercial airlines ban transport of non-human primates to laboratories, pressurizing airlines by orchestrated e-mail campaigns, Facebook postings and phone calls. No North American carriers currently transport primates to research institutions; United Airlines announced earlier this year that it was halting its transport of research primates. Only four airlines currently allow the transport of primates on their flights: Air France, China Eastern Airlines, Philippine Airlines and Vietnam Airlines (http://tinyurl.com/a46epcc). Bowing to pressure, the last ferry company that was willing to transport research rodents into the UK stopped doing so in 2012.

These insidious efforts are successful because they rely on the assumption that chipping away slowly at the ability to do animal research will go unnoticed, until research becomes too arduous and limitations too great to get substantial work done. Many of the concerns raised by animal rights activists, such as the availability of alternative methods and reducing the number and suffering of animals, are already incorporated into existing animal research protocols; to gain ethical approval for studies, researchers need to demonstrate that there is no alternative to the use of animals and that every attempt is made to keep the number of animals and their suffering to a minimum. Scientists and their friends need to urgently make a loud and persuasive case to defend the use of animals in research and to speak up against such campaigns. They need to mobilize the millions of patients and families that have been affected by disease and protest this impediment to research as publicly and as dramatically as the animal activists have done. Such tactics have been successful in turning back the tide of animal rights extremism in the UK, where patients speaking out in favor of animal research have made a very persuasive public case for why such work needs to continue (http://tinyurl.com/d6jqmpg). We also need to educate the public on what is already being done to reduce animal use and to ensure their ethical treatment. Silently enduring these campaigns ensures a stagnation of biomedical research and guarantees that precious animal lives will be wasted. 